\title{
Semismoothness in parametrized quasi-variational inequalities
}

\author{
Jiři V. Outrata \\ Institute of Information Theory and Automation \\ Academy of Sciences of the Czech Republic \\ Pod vodárenskou věži 4, 18208 Prague, Czech Republic. \\ e-mail: outrata@utia.cas.cz
}

\begin{abstract}
The paper deals with stability and sensitivity analysis of a class of parameter-dependent quasi-variational inequalities. By using the stability theory of Robinson for generalized equations, we compute the directional derivative and the generalized Jacobian of the map which assigns to the parameter the (locally unique) solution of the quasi-variational inequality. This enables to prove that this map is in fact semismooth and, consequently, to apply various methods of nonsmooth analysis to the numerical treatment of an interesting class of equilibrium problems.
\end{abstract}

\section{Keywords}

Quasi-variational inequality, directional derivative, generalized Jacobian, semismoothness.

\section{INTRODUCTION}

Quasi-Variational Inequalities (QVIs), introduced in the seventies in connection with some stochastic impulse control problems, represent an important class of implicitly constrained variational inequalities. They provide a useful tool for modelling of various complicated equilibria (Baiocchi and Capelo, 1984). So far, the basic existence and uniqueness questions have been answered and the mathematicians have turned among others to the stability and sensitivity of their solutions with respect to parameter (Kyparisis and Ip, 1992, Kočvara and Outrata, 1994). In the present paper we use the stability theory of Robinson for generalized equations (GEs) to ensure the lipschitzian behaviour of the (locally unique) solution of our QVI with respect to the parameter and to compute the appropriate directional derivative (Section 2). Then we turn our attention to the generalized Jacobians of this mapping, whereby we use the sensitivity result of Kyparisis (1990). The comparison of directional derivatives and generalized Jacobians enables to prove the main result - the semismoothness of the investigated mapping. This is done in Section 3. The semismoothness property plays a crucial role in a number of important numerical approaches of nonsmooth analysis, in particular in bundle methods of nonsmooth optimization (Schramm and Zowe, 1992) and in a nonsmooth variant of the Newton method 
(Qi, 1993). In Section 4 we show, how these methods may be applied to the numerical solution of

- inverse problems generated by parameter-dependent QVIs, and

- optimization problems with QVIs as constraints.

The paper presumes a certain basic knowledge of some concepts of nonsmooth analysis. For reader's convenience we state here at least the definition of a semismooth map.

Definition 1 (Qi and Sun, 1993). An operator $F\left[\mathbb{R}^{n} \rightarrow \mathbb{R}^{m}\right]$ is said to be semismooth at $x_{0} \in \mathbb{R}^{n}$ if it is Lipschitz near $x_{0}$ and

$\lim _{\substack{v \in \& F\left(x_{0}+t h^{\prime}\right) \\ h^{\prime} \rightarrow h_{s} \in t 0}}\{V h\}$

exists for each $h \in \mathbb{R}^{n}$.

The following notation is employed: $x^{i}$ is the $i$ th component of a vector $x \in \mathbb{R} R^{n}, E$ is the unit matrix and $B$ is the unit ball. If $\Omega$ is a closed convex set, then

$N_{\Omega}(x)=\left\{\begin{array}{l}\text { normal cone to } \Omega \text { at } x, \text { provided } x \in \Omega \\ \emptyset \text { otherwise. }\end{array}\right.$

\section{STABILITY ANALYSIS AND DIRECTIONAL DERIVATIVES}

Let $\mathcal{A}$ be an open set in $\mathbb{R}^{n}, F\left[\mathcal{A} \times \mathbb{R}^{n} \rightarrow \mathbb{R}^{m}\right]$ be a continuously differentiable operator and $g^{i}\left[\mathcal{A} \times \mathbb{R}^{m} \times \mathbb{R}^{m} \rightarrow \mathbb{R}\right], i=1,2, \ldots, s$, be twice continuously differentiable functions, convex in the third variable. With these data we define the parameter-dependent QVI:

For a given $x \in \mathcal{A}$ find a vector $y \in \Gamma(x, y)$ such that

$\left\langle F(x, y), y^{\prime}-y\right\rangle \geq 0 \quad$ for all $y^{\prime} \in \Gamma(x, y)$

thereby $\Gamma(x, y)=\left\{z \in \mathbb{R}^{m} \mid g^{i}(x, y, z) \leq 0, i=1,2, \ldots s\right\}$.

Let $S$ be the (set-valued) map assigning to $x \in \mathcal{A}$ the (sets of) solutions of (1). To analyse the local behaviour of this map, we fix a parameter $x_{0} \in \mathcal{A}$ and assume that $y_{0} \in S\left(x_{0}\right)$. Throughout the first two sections it is supposed that

(LI) the partial gradients $\nabla_{3} g^{i}\left(x_{0}, y_{0}, y_{0}\right)$ for $i \in I\left(x_{0}, y_{0}\right):=\{j \in\{1,2, \ldots, s\} \mid$ $\left.g^{j}\left(x_{0}, y_{0}, y_{0}\right)=0\right\}$ are linearly independent.

It is well-known that the QVI (1) can be equivalently written as a nonsmooth equation

$y=\operatorname{Proj}_{\Gamma(x, y)}(y-F(x, y))$.

Evidently, under a suitable constraint qualification, $z=\operatorname{Proj}_{\Gamma\left(x_{0}, y_{0}\right)}\left(y_{0}-F\left(x_{0}, y_{0}\right)\right)$ if and only if there exists a Karush-Kuhn-Tucker (KKT) vector $\lambda_{0} \in R_{+}^{s}$ such that

$0 \in\left[\begin{array}{c}z-y_{0}+F\left(x_{0}, y_{0}\right)+\sum_{i=1}^{s} \lambda_{0}^{i} \nabla_{3} g^{i}\left(x_{0}, y_{0}, z\right) \\ -G\left(x_{0}, y_{0}, z\right)\end{array}\right]+\left[\begin{array}{c}0 \\ N_{\boldsymbol{R}_{+}^{s}}^{s}\left(\lambda_{0}\right)\end{array}\right]$ 
thereby

$G(x, y, z)=\left[\begin{array}{c}g^{1}(x, y, z) \\ g^{2}(x, y, z) \\ \vdots \\ g^{s}(x, y, z)\end{array}\right]$.

Hence it is clear that under (LI), $y_{0} \in S\left(x_{0}\right)$ if and only if $y_{0}$ solves together with a $\lambda_{0} \in \mathbb{R}_{+}^{s}$ the GE

$0 \in\left[\begin{array}{c}\mathcal{L}\left(x_{0}, y, \lambda\right) \\ -G\left(x_{0}, y, y\right)\end{array}\right]+\left[\begin{array}{c}0 \\ N_{\mathbf{R}_{+}^{s}}(\lambda)\end{array}\right]$

where $\mathcal{L}(x, y, \lambda)=F(x, y)+\sum_{i=1}^{s} \lambda^{i} \nabla_{3} g^{i}(x, y, y)$ is the Lagrangian related to the QVI (1). Due to (LI) to each $y_{0} \in S\left(x_{0}\right)$ the KKT vector $\lambda_{0}$ is uniquely determined. Therefore we may introduce another index set

$J\left(x_{0}, y_{0}\right):=\left\{i \in I\left(x_{0}, y_{0}\right) \mid \lambda_{0}^{i}>0\right\}$.

The constraints corresponding to $i \in I\left(x_{0}, y_{0}\right), i \in J\left(x_{0}, y_{0}\right)$ and $i \in I\left(x_{0}, y_{0}\right) \backslash J\left(x_{0}, y_{0}\right)$ are termed active, strongly active and semiactive at $\left(x_{0}, y_{0}\right)$, respectively. To shorten the notation, for an index set $K \subset\{1,2, \ldots, s\}$ and a vector $d \in \mathbb{R}^{s}, d_{K}$ denotes the subvector composed from the components $d^{i}, i \in K$. Analogously, for a matrix $D$ with $s$ rows, $D_{K}$ is the submatrix composed from the rows $D^{i}, i \in K$ and $[D]_{n}$ is the submatrix with the rows $D^{i}, i=1,2, \ldots, n(n \leq s)$. At the index sets $I(x, y), J(x, y)$ the arguments will be dropped whenever it cannot lead to a confusion.

The theory of Robinson (1980) leads to the following important statement, in which $\Psi$ is the map assigning to $x \in \mathcal{A}$ the (sets of) solutions of the GE (4).

Proposition 1. Let

$Q:=\left[\begin{array}{cc}\nabla_{2} \mathcal{L}\left(x_{0}, y_{0}, \lambda_{0}\right) & \left(\nabla_{3} G\left(x_{0}, y_{0}, y_{0}\right)\right)^{\mathrm{T}} \\ -\nabla_{2} G\left(x_{0}, y_{0}, y_{0}\right)-\nabla_{3} G\left(x_{0}, y_{0}, y_{0}\right) & 0\end{array}\right]$

and consider the linear GE

$\xi \in Q\left[\begin{array}{l}v \\ u\end{array}\right]+\left[\begin{array}{c}0 \\ N_{\boldsymbol{R}_{+}^{s}}(u)\end{array}\right]$.

Assume that the GE (4) is strongly stable at $\left(x_{0}, y_{0}, \lambda_{0}\right)$, i. e. the GE (5) possesses a unique solution $(v, u) \in \mathbb{R}^{m} \times \mathbb{R}_{+}^{s}$ for each $\xi \in \mathbb{R}^{m+s}$, cf. Kyparisis (1990). Then there exist neighbourhoods $\mathcal{O}$ of $x_{0}, \mathcal{M}$ of $y_{0}$ and $\mathcal{N}$ of $\lambda_{0}$ such that the map $\widetilde{\Psi}(\cdot):=\Psi(\cdot) \cap(\mathcal{M} \times \mathcal{N})$ is single-valued and Lipschitz on $\mathcal{O}$.

In such a case, evidently, $\widetilde{\Psi}$ splits on $\mathcal{O}$ into two operators $\widetilde{S}$ and $\widetilde{\Lambda}$ which assign to $x \in \mathcal{O}$ the $y$ - and $\lambda$-components of the solution $\widetilde{\Psi}(x)$ to the GE (4).

By using results from the theory of complementarity problems, it has been proved in Robinson (1980) that the condition of Prop. 1 is equivalent to the following requirement: The matrix

$$
R=\left[\begin{array}{cc}
\nabla_{2} \mathcal{L}\left(x_{0}, y_{0}, \lambda_{0}\right) & \left(\nabla_{3} G_{J}\left(x_{0}, y_{0}, y_{0}\right)\right)^{\mathrm{T}} \\
-\nabla_{2} G_{J}\left(x_{0}, y_{0}, y_{0}\right)-\nabla_{3} G_{J}\left(x_{0}, y_{0}, y_{0}\right) & 0
\end{array}\right]
$$


is nonsingular and its Schur complement to

$Q_{\text {red }}=\left[\begin{array}{cc}\nabla_{2} \mathcal{L}\left(x_{0}, y_{0}, \lambda_{0}\right) & \left(\nabla_{3} G_{I}\left(x_{0}, y_{0}, y_{0}\right)\right)^{\mathbf{T}} \\ -\nabla_{2} G_{I}\left(x_{0}, y_{0}, y_{0}\right)-\nabla_{3} G_{I}\left(x_{0}, y_{0}, y_{0}\right) & 0\end{array}\right]$

is a $P$-matrix (has positive principal minors). However, it is not easy to ensure these properties in terms of the original problem data. In fact, we have succeeded to get such a statement only in the case if (1) corresponds to an implicit complementarity problem (ICP), i. e. if $s=m$ and the constraint functions $g^{i}$ have a special structure

$g^{i}(x, y, z)=\varphi^{i}(x, y)-z^{i}, \quad i=1,2, \ldots, m$.

Proposition 2. Let $\nabla_{2} F\left(x_{0}, y_{0}\right)$ be positive definite and $\nabla_{2} \Phi\left(x_{0}, y_{0}\right)\left(\nabla_{2} F\left(x_{0}, y_{0}\right)\right)^{-1}$ be negative semidefinite, whereby

$\Phi(x, y)=\left[\begin{array}{c}\varphi^{1}(x, y) \\ \varphi^{2}(x, y) \\ \vdots \\ \varphi^{m}(x, y)\end{array}\right]$.

Then the assertion of Prop. 1 holds true.

Proof. The GE (5) attains the form

$\left[\begin{array}{l}\xi_{1} \\ \xi_{2}\end{array}\right] \in\left[\begin{array}{cc}\nabla_{2} F\left(x_{0}, y_{0}\right) & -E \\ -\nabla_{2} \Phi\left(x_{0}, y_{0}\right)+E & 0\end{array}\right]\left[\begin{array}{l}v \\ u\end{array}\right]+\left[\begin{array}{c}0 \\ N_{R_{+}^{m}}(u)\end{array}\right]$,

where $\xi=\left(\xi_{1}, \xi_{2}\right) \in \mathbb{R}^{m} \times \mathbb{R}^{m}$. As $\nabla_{2} F\left(x_{0}, y_{0}\right)$ is positive definite, it is nonsingular and its inverse is also positive definite. Thus, (7) amounts to the equation

$v=\left(\nabla_{2} F\left(x_{0}, y_{0}\right)\right)^{-1}\left(\xi_{1}+u\right)$

and the linear complementarity problem

$\xi_{2}+\left(\nabla_{2} \Phi\left(x_{0}, y_{0}\right)-E\right)\left(\nabla_{2} F\left(x_{0}, y_{0}\right)\right)^{-1} \xi_{1} \in\left(E-\nabla_{2} \Phi\left(x_{0}, y_{0}\right)\right)\left(\nabla_{2} F\left(x_{0}, y_{0}\right)\right)^{-1} u+N_{R_{+}^{m}}(u)$.

Under the imposed assumptions this linear complementarity problem possesses a unique solution $u$ for each $\xi_{1}, \xi_{2}$ and then also the $v$-component of the solution of (7) is uniquely determined. In this way the suppositions of Prop. 1 have been verified.

Henceforth it is assumed that the GE (4) corresponding to the original QVI fulfills the assumption of Prop. 1, whereby we are aware that the verification may be quite complicated. Then the results of Robinson $(1980,1991)$ imply also the directional differentiability of the map $\widetilde{S}$ at $x_{0}$. On denoting by $\widetilde{S}^{\prime}\left(x_{0} ; h\right)$ this directional derivative in the direction 
$h \in \mathbb{R}^{n}, \widetilde{S}^{\prime}\left(x_{0} ; h\right)=v$ satisfies with a vector $u \in \mathbb{R}^{s}$ the system of equations and inequalities

$$
\begin{aligned}
& \nabla_{2} \mathcal{L}\left(x_{0}, y_{0}, \lambda_{0}\right) v+\left(\nabla_{3} G_{I}\left(x_{0}, y_{0}, y_{0}\right)\right)^{\mathrm{T}} u_{I}=-\nabla_{1} \mathcal{L}\left(x_{0}, y_{0}, \lambda_{0}\right) h \\
& {\left[\nabla_{3} G_{J}\left(x_{0}, y_{0}, y_{0}\right)+\nabla_{2} G_{J}\left(x_{0}, y_{0}, y_{0}\right)\right] v }=-\nabla_{1} G_{J}\left(x_{0}, y_{0}, y_{0}\right) h \\
& {\left[\nabla_{3} G_{I \backslash J}\left(x_{0}, y_{0}, y_{0}\right)+\nabla_{2} G_{I \backslash J}\left(x_{0}, y_{0}, y_{0}\right)\right] v } \leq-\nabla_{1} G_{I \backslash J}\left(x_{0}, y_{0}, y_{0}\right) h \\
& u^{i}=0 \text { for } i \notin I\left(x_{0}, y_{0}\right) \\
& u_{I \backslash J} \geq 0 \\
&\left(\left\langle\nabla_{1} g^{i}\left(x_{0}, y_{0}, y_{0}\right), h\right\rangle+\left\langle\nabla_{2} g^{i}\left(x_{0}, y_{0}, y_{0}\right)+\nabla_{3} g^{i}\left(x_{0}, y_{0}, y_{0}\right), v\right\rangle\right) u^{i}=0 \text { for } i \in I\left(x_{0}, y_{0}\right) \backslash J\left(x_{0}, y_{0}\right) .
\end{aligned}
$$

This system will be used in the next section.

\section{GENERALIZED JACOBIANS AND SEMISMOOTHNESS}

The local behaviour of the map $\widetilde{S}$ near $x_{0}$ may also be characterized by so-called generalized Jacobian, cf. Clarke (1983). Consider the family $\mathcal{P}\left(I\left(x_{0}, y_{0}\right) \backslash J\left(x_{0}, y_{0}\right)\right)$ of all subsets of $I\left(x_{0}, y_{0}\right) \backslash J\left(x_{0}, y_{0}\right)$ and denote the single sets from this family by $T_{i}$, where $i$ runs through a suitably chosen index set $\mathbb{K}\left(x_{0}, y_{0}\right)$. Denote for $i \in \mathbb{K}\left(x_{0}, y_{0}\right)$

$$
\begin{aligned}
D_{J \cup T_{i}}\left(x_{0}, y_{0}, \lambda_{0}\right) & =\left[\begin{array}{cc}
\nabla_{2} \mathcal{L}\left(x_{0}, y_{0}, \lambda_{0}\right) & \nabla_{3} G_{J \cup T_{i}}\left(x_{0}, y_{0}, y_{0}\right)^{\mathrm{T}} \\
-\nabla_{2} G_{J \cup T_{i}}\left(x_{0}, y_{0}, y_{0}\right)-\nabla_{3} G_{J \cup T_{i}}\left(x_{0}, y_{0}, y_{0}\right) & 0
\end{array}\right], \\
B_{J \cup T_{i}}\left(x_{0}, y_{0}, \lambda_{0}\right) & =\left[\begin{array}{c}
-\nabla_{1} \mathcal{L}\left(x_{0}, y_{0}, \lambda_{0}\right) \\
\nabla_{1} G_{J \sqcup T_{i}}\left(x_{0}, y_{0}, y_{0}\right)
\end{array}\right] .
\end{aligned}
$$

From the analysis below Prop. 1 it is clear that each matrix $D_{J \cup T_{i}}$ is nonsingular ( $R$ is nonsingular and each submatrix of a $P$-matrix is again a $P$-matrix and hence nonsingular). Proposition 3. The generalized Jacobian of the map $\widetilde{S}$ at $x_{0}$, denoted $\partial \widetilde{S}\left(x_{0}\right)$, admits an upper estimate

$\partial \widetilde{S}\left(x_{0}\right) \subset \operatorname{conv}\left\{\left[\left(D_{J \cup T_{i}}\left(x_{0}, y_{0}, \lambda_{0}\right)\right)^{-1} B_{J \cup T_{i}}\left(x_{0}, y_{0}, \lambda_{0}\right)\right]_{m} \mid i \in \mathbb{I K}\left(x_{0}, y_{0}\right)\right\}$.

Proof. The proof is essentially given in Outrata (1994). However, as our assumptions are slightly weaker, we repeat briefly its main idea. The strong stability implies due to Kyparisis (1990) the existence of a neighbourhood $\mathcal{U}$ of $x_{0}$ such that whenever $x \in \mathcal{U}$ and the maps $\widetilde{S}, \widetilde{\Lambda}$ are differentiable at $x$, then there exist an index $i \in \mathbb{K}\left(x_{0}, y_{0}\right)$ such that

$D_{J \cup T_{i}}(x, \widetilde{S}(x), \widetilde{\Lambda}(x))\left[\begin{array}{c}\nabla \widetilde{S}(x) \\ \nabla \widetilde{\Lambda} J \cup T_{i}(x)\end{array}\right]=B_{J \cup T_{i}}(x, \widetilde{S}(x), \widetilde{\Lambda}(x))$

and the matrix $D_{J u r_{i}}(x, \widetilde{S}(x), \widetilde{\Lambda}(x))$ is nonsingular. By the definition of the generalized Jacobian we just need to construct all possible limits of such derivatives if $x$ tends to $x_{0}$. However, all these limits belong to the set $\left\{\left(D_{J \cup T_{i}}\left(x_{0}, y_{0}, \lambda_{0}\right)\right)^{-1} B_{J \cup T_{i}}\left(x_{0}, y_{0}, \lambda_{0}\right) \mid i \in \mathbb{K}\left(x_{0}, y_{0}\right)\right\}$ and so incl. (9) follows.

We are now ready to state the main result: 
Theorem 4. The map $\widetilde{S}$ is semismooth at $x_{0}$.

Proof. By Qi and Sun (1993) it suffices to show that

$\widetilde{S}^{\prime}\left(x_{0} ; h\right)=\lim _{\substack{v \in \partial \widetilde{S}\left(x_{0}+t h\right) \\ i 10}}\{V h\}$

and that the convergence is uniform for all $h \in B$. Assume by contradiction that there exists an $\varepsilon>0$, sequences $\left\{h_{i}\right\} \subset B, t_{i} \downarrow 0$ and matrices $V_{i} \in \partial \widetilde{S}\left(x_{0}+t_{i} h_{i}\right)$ for which

$\left\|V_{i} h_{i}-\widetilde{S}^{\prime}\left(x_{0} ; h_{i}\right)\right\|>\varepsilon$ for $i=1,2, \ldots$

As the generalized Jacobian mapping is uniformly compact and $\left\{h_{i}\right\} \subset B$, we can select convergent subsequences $h_{i^{\prime}} \rightarrow h, V_{i^{\prime}} \rightarrow V$, whereby $h \in B$ and $V \in \partial \widetilde{S}\left(x_{0}\right)$ (due to the closedness of $\partial \widetilde{S})$. The map $\widetilde{S}^{\prime}\left(x_{0} ; \cdot\right)$ is lipschitzian and so

$\left\|V h-\widetilde{S}^{\prime}\left(x_{0} ; h\right)\right\| \geq \varepsilon$.

From Prop. 3 we know that

$V=\sum_{\ell \in K\left(x_{0}, y_{0}\right)} \alpha_{\ell} P^{\ell}\left(x_{0}\right)$

where $\alpha_{\ell} \geq 0, \sum_{\ell \in \boldsymbol{K}\left(x_{0}, y_{0}\right)} \alpha_{\ell}=1$, and $P^{\ell}\left(x_{0}\right)$ consists from the first $m$ rows of the matrix $\left(D_{J \cup T_{\ell}}\left(x_{0}, y_{0}, \lambda_{0}\right)\right)^{-1} B_{J \cup T_{\ell}}\left(x_{0}, y_{0}, \lambda_{0}\right)$. Ineq. (10) implies the existence of a $j \in \mathbb{K}\left(x_{0}, y_{0}\right)$ such that $\alpha_{j}>0$ and $P^{j}\left(x_{0}\right) h \neq \widetilde{S}^{\prime}\left(x_{0} ; h\right)$. However, by (8) this is possible only if

$\left[\nabla_{3} g^{k}\left(x_{0}, y_{0}, y_{0}\right)+\nabla_{2} g^{k}\left(x_{0}, y_{0}, y_{0}\right)\right] S^{\prime}\left(x_{0} ; h\right)<-\nabla_{1} g^{k}\left(x_{0}, y_{0}, y_{0}\right) h$

for some $k \in T_{j}$. Let $M\left(x_{0}, y_{0}\right)$ denote the subset of $I\left(x_{0}, y_{0}\right) \backslash J\left(x_{0}, y_{0}\right)$ consisting of those indices $k$ for which ineq. (11) holds. Furthermore, let $\widetilde{K}\left(x_{0}, y_{0}\right)$ be an index set specifying the subsets of $\mathcal{P}\left(I\left(x_{0}, y_{0}\right) \backslash J\left(x_{0}, y_{0}\right) \backslash M\left(x_{0}, y_{0}\right)\right)$ in the same way as $\mathbb{K}\left(x_{0}, y_{0}\right)$ specifies the subsets of $\mathcal{P}\left(I\left(x_{0}, y_{0}\right) \backslash J\left(x_{0}, y_{0}\right)\right)$. Clearly, $P^{\ell}\left(x_{0}\right) h=\widetilde{S}^{\prime}\left(x_{0} ; h\right)$ for all $\ell \in \widetilde{\mathbb{K}}\left(x_{0}, y_{0}\right)$.

Due to the continuity of $S^{\prime}\left(x_{0} ; \cdot\right)$, inequalities (11) imply that

$g^{k}\left(x_{0}+t_{i^{\prime}} h_{i^{\prime}}, \widetilde{S}\left(x_{0}+t_{i^{\prime}} h_{i^{\prime}}\right), \widetilde{S}\left(x_{0}+t_{i^{\prime}} h_{i^{\prime}}\right)\right)<0 \quad$ for $k \in M\left(x_{0}, y_{0}\right)$

whenever $i^{\prime}$ is sufficiently large. Therefore, by Prop. 3 , for such $i^{\prime}$ one has

$V_{i^{\prime}} \in \operatorname{conv}\left\{P^{\ell}\left(x_{0}+t_{i^{\prime}} h_{i^{\prime}}\right) \mid \ell \in \widetilde{\mathbb{K}}\left(x_{0}, y_{0}\right)\right\}$.

If we now take also ineq. (10) into account, we observe that there exists a natural number $n_{0}$ such that for all $i^{\prime}>n_{0}$

$\left\|V_{i^{\prime}} h-\widetilde{S}^{\prime}\left(x_{0} ; h\right)\right\|>\frac{\varepsilon}{2}$,

where $V_{i^{\prime}}$ is given by (12). However, this is impossible due to the continuity of the maps $x \mapsto P^{\ell}(x), \ell \in \widetilde{K}\left(x_{0}, y_{0}\right)$, in a neighbourhood of $x_{0}$. The assertion has been proved. 
Remark. If the functions $g^{i}$ do not depend on $y$, the QVI (1) becomes just an ordinary parameter-dependent variational inequality and the above result reduces to (Outrata and Zowe, 1995, Prop. 4).

\section{APPLICATIONS}

The results of the previous sections may be applied in the numerical solution of different equilibrium problems. Let $m=n$ and consider the inverse problem to the QVI (1):

For a given $y_{0} \in \mathbb{R}^{m}$ find $x \in \mathcal{A}$ such that $y_{0} \in S(x)$. Assume that this problem possesses a solution $x_{0}$ and that the (LI) constraint qualification as well as the strong stability from Section 2 hold.

Then one could apply the nonsmooth Newton variant of Qi (1993) to the numerical solution of this problem. The Newton iteration attains the form

$x_{k+1}=x_{k}-V_{k}^{-1}\left(\widetilde{S}\left(x_{k}\right)-y_{0}\right), \quad k=1,2, \ldots$,

where $V_{k} \in \partial_{B} \widetilde{S}\left(x_{k}\right):=\left\{\lim \nabla \widetilde{S}(y) \mid y \rightarrow x_{k}, \widetilde{S}\right.$ is differentiable at $\left.y\right\}$.

Proposition 5. Assume that all matrices from $\partial_{B} \widetilde{S}\left(x_{0}\right)$ are nonsingular. Then the method (13) is locally superlinearly convergent.

Proof. As $\widetilde{S}$ is semismooth at $x_{0}$, it is an easy consequence of (Qi, 1993, Thm. 3.1).

Of course, under the above mild assumptions we may need a starting point very close to the solution. The reason is that even if the neighbourhood $\mathcal{O}$ (on which $\widetilde{S}$ is defined) is large enough, we actually need the strong stability on the whole $\mathcal{O}$ to dispose with the appropriate estimates of $\partial_{B} \widetilde{S}\left(x_{k}\right)$. In fact, at nonsmooth points of $\widetilde{S}$ the appropriately modified incl. (9) does not directly lead to the desired matrix $V_{k}$, but such points are met extremely rarely during the iteration process. To illustrate this sort of problems, we can take a discretized obstacle problem with a membrane (plate) and a complient obstacle (Kočvara and Outrata, 1994). The task is to compute the load imposed on the membrane (plate) such that a desired deflection is achieved.

Another class of equilibrium problems, where the results of previous sections may well be utilized, are the optimization problems with quasi-variational inequality constraints. Assume that $S$ admits a lipschitzian selection $\widetilde{S}$, defined on a given compact subset of $\mathcal{A}$, denoted $U_{\text {ad. }}$. Let $J\left[\mathcal{A} \times \mathbb{R}^{m} \rightarrow \mathbb{R}\right]$ be a continuously differentiable objective and consider the nonsmooth optimization problem

$$
\begin{aligned}
\operatorname{minimize} & J(x, y) \\
\text { subject to } & y=\widetilde{S}(x) \\
& x \in U_{\mathbf{a d}}
\end{aligned}
$$

This problem clearly possesses a solution. It amounts to the minimization of the locally Lipschitz composite function $\Theta(x):=J(x, \widetilde{S}(x))$ over $U_{\text {ad }}$ to which a suitable bundle method may be applied. However, one has to ensure that $\Theta$ is at least weakly semismooth (Schramm and Zowe, 1992) and be able to compute at each $x \in U_{\text {ad }}$ one arbitrary vector 
from $\partial \Theta(x)$. As $J$ is continuously differentiable, the semismoothness of $\widetilde{S}$ directly implies the semismoothness of $\Theta$. Moreover, knowing a matrix from $\partial \widetilde{S}(x)$, one can easily compute a desired vector from $\partial \Theta(x)$ by the standard chain rule. Thus, we merely need to require the satisfaction of the (LI) constraint qualification at all pairs $(x, \widetilde{S}(x))$ and the appropriate strong stability assumption for all $x$ from an open set containing $U_{\text {ad }}$. A distinguished example representing this class of problems is the design of robot manipulators, whereby we optimize the stress distribution of each gripper. The constraining QVI results from the reciprocal formulation of the appropriate contact problem with Coulomb friction.

\section{REFERENCES}

Baiocchi, C. and Capelo, A. (1984) Variational and Quasi-Variational Inequalities. Applications to Free Boundary Problems. Wiley, New York.

Clarke, F. H. (1983) Optimization and Nonsmooth Analysis. Wiley, New York.

Kočvara, M. and Outrata J.V. (1994) On optimization of systems governed by implicit complementarity problems. Numerical Functional Analysis and Optimization, 15, 86987.

Kyparisis, J. (1990) Solution differentiability for variational inequalities. Mathematical Programming, 48, 285-301.

Kyparisis, J. and Ip, Ch. M. (1992) Solution behaviour for parametric implicit complementarity problems. Mathematical Programming, 56, 65-70.

Outrata, J.V. (1994) On optimization problems with variational inequality constraints. SIAM J. Optimization, 4, 340-57.

Outrata, J.V. and Zowe, J. (1995) A Newton method for a class of quasi-variational inequalities. Computational Optimization and Applications, 4, 5-21.

Qi, L. (1993) Convergence analysis of some algorithms for solving nonsmooth equations. Mathematics of Operations Research, 18, 227-44.

Qi, L. and Sun, J. (1993) A nonsmooth version of Newton's method. Mathematical Programming, 58, 353-67.

Robinson, S. M. (1980) Strongly regular generalized equations. Mathematics of Operations Research, 5, 43-62.

Robinson, S. M. (1991) An implicit function theorem for a class of nonsmooth functions. Mathematics of Operations Research, 16, 282-309.

Schramm, H. and Zowe, J. (1992) A version of the bundle idea for minimizing a nonsmooth function: Conceptual idea, convergence analysis, numerical results. SIAM J. Optimization, 2, 121-252. 